\title{
Memory Limited Trust Public Opinion Evolution Model In Accelerated Growth HK Network
}

\author{
Zhang Jingshan ${ }^{1}$, Ma Baohua ${ }^{2}$, Sun Shibao ${ }^{3}$ and Zhang Yanan ${ }^{4}$ \\ ${ }^{1}$ College of Information Engineering, Henan University of Science and Technology, \\ Luoyang Henan 471023, China \\ ${ }^{2}$ Science and Technology Department, Henan University of Science and Technology, \\ Luoyang Henan 471023, China \\ ${ }^{3}$ College of Information Engineering, Henan University of Science and Technology, \\ Luoyang Henan 471023, China \\ ${ }^{4}$ College of Information Engineering, Henan University of Science and Technology, \\ Luoyang Henan 471023, China
}

\begin{abstract}
To research the influence of individual subjectivity for opinion evolution, improved classical bounded confidence model, introduced firmness degree, trust degree and memory length, proposed a new method for measuring the influence among individuals. In consideration of the accelerated growth characteristics of real networks, established a network public opinion evolution model with memory mechanism based on accelerated growth HK network; studied the influence of trust threshold and memory length on public opinion evolution. The results show that, the value of trust threshold is inversely proportional to the final number of opinion clusters; memory length is proportional to the time of group opinions convergence.

Keywords: Network opinion, Accelerated growth, Memory, limitary.
\end{abstract}

\section{Introduction}

Internet public opinion is a collection of people's cognitive, attitude, emotional and behavioral tendencies towards the event generated by the stimulation of the incident through the Internet.[1]With the development of the Internet, the influence of Internet public opinion on society and politics is becoming more and more prominent, and the research of network public opinion has received more and more attention. Analyzing and mastering the evolution of public opinion, to provide reference for decision makers to guide public opinion, to reduce the negative impact of network public opinion. Analyzing and mastering the law of public opinion evolution can provide reference for decision makers to guide and control the public opinion, and reduce the negative impact of network public opinion.

At present, the research of network public opinion evolution mainly includes two types: one is the empirical research, through the analysis of public opinion research data of real network evolution factors; the other is modeling and simulation, using model to simulate the interactive process of individual views, revealing the characteristics and laws of public opinion evolution. Due to the relevant technology of empirical research is not mature, simulation modeling is still the main method of network public opinion research. According to the different expression methods of individual views, the evolution model of public opinion can be divided into two categories: One kind is the discrete view model, typical representative is the Sznajd model[2] and the election model; The other is a continuous view model, the Deffaunt model [3] and Hegselmann-Krause (HK) model are widely used[4].These two models express individual opinions in a continuous range, and introduce the concept of bounded confidence, establish a continuous view limited trust model, to reflect the fact that people's views are not quite distinct from each other.

Using the social network to represent the relationship among individuals in limited trust model, individual do not blindly follow other individual opinions, communicate only when their opinions are similar. Considering the influence of individuals, the threshold of trust and the strategy of individual, a view evolution model of high impact priority limited trust is proposed in paper [5]. Paper [6] think that the impact of population and trust is a process from quantitative to qualitative change, proposed a evolution model based on continuous function of public opinion influence. A heterogeneous finite trust model is proposed in paper[7], which discusses the influence of sudden events and different levels of trust on the public opinion evolution.HK model is extended in Paper[8], and 
the model based on the limited impact hypothesis is proposed.

In the construction of trust relationship between individuals, the above limited trust model mainly considered opinion similarity, individual influence and relationship between individuals, without taking into account the effect of memory on trust relationship in the process of individual communication. Plenty of research have shown that the real network has the characteristic of accelerating growth, and an accelerated growth HK model is proposed in paper[9].According to the interactive features of the Internet users, a network public opinion evolution model in the accelerated growth HK network is proposed, which is based on the comprehensive consideration of interaction between users, trust threshold and individual memory strategies, in order to provide the basis for the research of network public opinion.

\section{Limited trust model with memory}

When constructing the trust relationship in the process of interaction, mainstream evolution models based on the trust boundary constraints consider opinion similarity, individual influence and individual relationship, without considering the effect of memory on trust relationship in the process of individual communication. In the evolution process of the real network public opinion, individual does not refer to the opinions of all other individuals in the network, but refer to opinion of person who has contact with and has similar views.

Considering the influence of memory on the evolution of public opinion, use $\mathrm{M}$ to represent the length of memory. In the length of memory $\mathrm{M}$, when the individual neighbors is not in the trust threshold $\varepsilon$ will retain the memory of distrust. The interaction between individuals will change when the view is updated. The individual will disconnect neighbors with different opinions in a memory length $\mathrm{M}$, do not exchange views whether their opinions are close. However, memory is only alive in a period of time, at the end of each memory length the memory of distrust will be forgotten, disconnect connection between individuals will be reconnected, individual determine whether or not to exchange opinions according to the point of view.

According to the characteristics of user communication, a memory limited trust public opinion evolution model is proposed. The process of opinion update can be divided into several steps: First, count trust set of node according to the difference of opinion; Then, record those individuals which are not in the threshold of trust and disconnect from the individual in the current memory length; The individual's opinion at the next moment is calculated by current individual opinion and other individuals' opinions in trust set. Reconnect the disconnected nodes at the end of each memory length $M$, and then repeat the above steps until group opinion tend to be stable.

\subsection{Construct the initial network model}

Construct the virtual social network of individual interaction according to the construction algorithm of accelerated growth HK model proposed in paper [9].Use $\mathrm{N}=\{1,2, \ldots, \mathrm{n}\}$ to represent individual in the network, $\mathrm{N}$ is the number of individuals, and the initial values of the individuals are uniformly distributed. $\mathrm{T}=\{0,1,2 \ldots\}$ represents the time of individual interaction; $\mathrm{X}(\mathrm{t})=\left[\mathrm{x}_{1}(\mathrm{t})\right.$, $\left.\mathrm{x}_{2}(\mathrm{t}), \cdots, \mathrm{x}_{\mathrm{n}}(\mathrm{t})\right]$ is a section of group view based on time $T$, initial values are uniformly distributed, $x_{i}(t)$ is the value of node $i$ at time of $t, x i(t) \in[0,1]$, set the $n \times n$ matrix $\mathrm{W}=\left[\omega_{\mathrm{ij}}\right]$ as the influence matrix. $\omega_{\mathrm{ij}} \in\{0,1\}, \omega_{\mathrm{ij}}=1$ indicates that there is a connection between $i$ and $j$, $\omega_{\mathrm{ij}}=0$ indicates that there is no connection between the nodes $\mathrm{i}$ and $\mathrm{j}$.

\subsection{Opinion evolution rule}

When individual changes opinion, only refer to opinion of individuals which have connection with itself and opinion distance is less than the threshold $\varepsilon$. Set individual set R, time $t$ and threshold $\varepsilon$, the trust set of individual i as shown in the formula (1).

$$
\mathrm{G}(\mathrm{i}, \mathrm{X}(\mathrm{t}), \varepsilon)=\left\{\mathrm{j}|| x_{i}(\mathrm{t})-x_{j}(\mathrm{t}) \mid \leq \varepsilon, \mathrm{j} \in \mathrm{R}(1)\right.
$$

The formula (1) shows that the trust set of $i$ is composed of individuals which are adjacent and view distance is less than the threshold $\varepsilon . \varepsilon$ is the key parameters affecting the size of the trust set, individuals exchange opinion only when the differences of their opinion is in the variable control range, and $\varepsilon \in(0,1)$.

In consideration of individual opinion is influenced by his current tendency and individuals in trust set, the definition of individual trust degree and self-firmness degree is as follows:

Trust degree $\omega_{i}(j, t)$ is the level of trust from $i$ to $j$ at the time of $\mathrm{t}$, and $\omega_{i}(j, t) \in(0,1)$. The trust function of $\mathrm{i}$ to its neighboring individuals at $\mathrm{t}$ is shown in formula (2).

$$
\omega_{i}(j, t)=\frac{k_{j}}{k_{i}+\sum_{<i, j>k_{j}}}, \quad j \in \mathrm{G} \quad(2)
$$

The individual trust degree from $i$ to $j$ is related to the degree of $i, j$ and the sum of individual degrees in its trust set. The higher the ratio of $j$ is, the higher the degree of trust from $\mathrm{i}$ to $\mathrm{j}$. In real network, the greater the number of fans, the greater the influence of individuals, the easier their views is trusted by others. Sum degree of node that has connection with $\mathrm{i}$ and view distance is less than $\varepsilon$ is represented by $\sum_{<i, j>} k_{j}$. 
Self-firmness degree $\omega_{i}(i, t)$ represents the probability that $\mathrm{i}$ adhere to current point of view at the time of $\mathrm{t}, \omega_{i}(i, t) \in(0,1]$, the calculation of i's firmness as shown in formula (3).

$$
\omega_{i}(i, t)=\frac{k_{i}}{k_{i}+\sum_{<i, j>} k_{j}}, j \in \mathrm{G}
$$

In real network, the more fans, the higher self-firmness degree of individual, the more difficult it is to be influenced by neighboring individuals.

Under certain circumstances, when view distance of all the neighbor nodes with $\mathrm{i}$ is greater than the trust threshold $\varepsilon$, trust set is empty, self-firmness degree $\omega_{i}(i, t)=1$, the next moment the current individual viewpoint will remain unchanged. It is obvious that $\omega_{i}(i, t)+\sum_{<i, j>} \omega_{i}(j, t)=$ 1 .

In the communication process of group views, the update rules of views are shown in formula (4):

$$
x_{i}(\mathrm{t}+1)=
$$

$$
\omega_{i}(i, t) x_{i}(\mathrm{t})+\sum_{j:\left|x_{i}(t)-x_{j}(t)\right|<\varepsilon} \omega_{i}(j, t) x_{j}(t), \quad j \in \mathrm{G}
$$

The greater the degree of individual $i$, the stronger the ability of $\mathrm{i}$ to keep opinion unchanged; the greater the degree of individual $\mathrm{j}$, the higher the trust degree of individual $\mathrm{i}$ to $\mathrm{j}$.

In formula (5) $G$ represents the trust set of $i$, because of the limited trust and memory, the network topology is in dynamic change, so trust set $\mathrm{G}$ is a time-varying set based on network topology and trust threshold in the evolution process of public opinion.

\section{Simulation experiment and result analysis}

\subsection{Model simulation algorithm}

The limited memory trust model based on accelerated growth HK network is established by using the simulation tool MatlabR2012b, the algorithm of the new model is as follows:

(1)Generate initial network. Set the number of network nodes $\mathrm{n}$, generate the initial network of group opinion evolution according to the accelerating growth network algorithm in this paper.

(2)Initialize parameters. Assign the initial values of all nodes in the network subject to $[0,1]$ uniform distribution by using Matlab random function. Set the trust threshold, memory length and simulation time length $t$.

(3)Opinion iteration. When $t=t+1$, calculate the individual trust set according to the formula (1) to, then the calculate self-firmness degree and individual trust degree within the trust set according to the formula (2) and (3), and calculate group opinion at $\mathrm{t}+1$ time in accordance with the formula (4), until the number of iterations is greater than the preset number $\mathrm{T}$.
(4)Output simulation results. Output opinion time varying curve of all nodes in the process of simulation experiment.

3.2 The influence of trust threshold on the opinion evolution

Considering too few nodes can't reflect the huge scale of the network and the excessive complexity of the nodes, set the number of nodes $\mathrm{N}=1000$. Accelerated growth index $\mathrm{r}=1.4$, memory length $\mathrm{M}=0$, trust threshold $\varepsilon$ for $0.05,0.1$, $0.2,0.25$ 。 The initial values of all individuals subject to $[0,1]$ uniform distribution, uniform distribution is a relatively reasonable assumption without any prior information. Evolution time $\mathrm{T}=50$, individual opinions are updated synchronously at each time step, experimental results are shown in Figure 1.

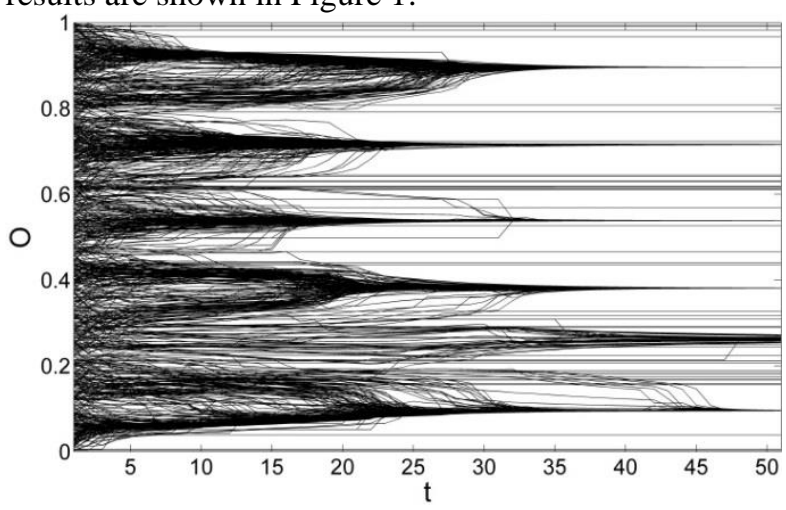

(a) threshold $\varepsilon=0.05$



(b) threshold $\varepsilon=0.1$






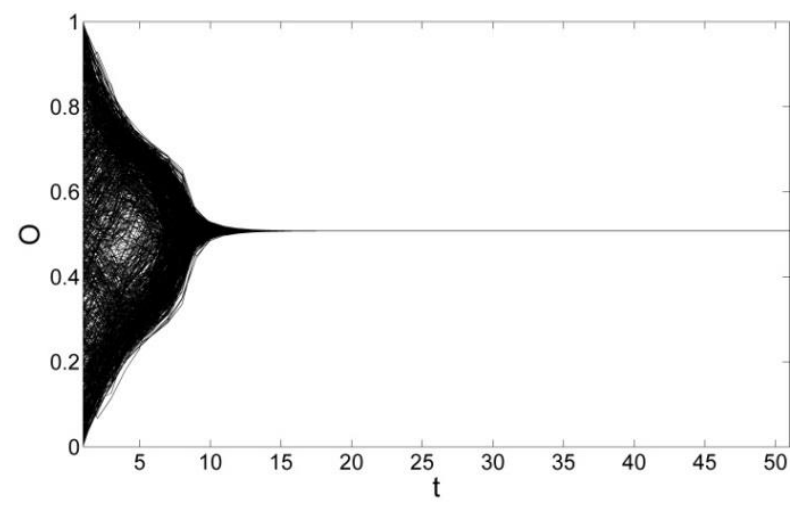

(d) threshold $\varepsilon=0.25$

Fig. 1 Effect of $\varepsilon$ on public opinion evolution

As shown in figure 1, the value of trust threshold $\varepsilon$ has great influence on the number of public view convergence under the new rule of network public opinion evolution. Public opinions split into multiple clusters, or be unanimous, group attitudes of opinion cluster finally reach stable state. When $\varepsilon=0.05$, public attitude is difficult to converge, and because of trust isolation in public opinion evolution, generate a large number of isolated nodes appear; When $\varepsilon=0.1$, public opinion converges around $\mathrm{t}=20$, generating 3 opinion clusters, along with some isolated nodes; When $\varepsilon=0.2$, public opinion converges around $\mathrm{t}=15$, generating 2 opinion clusters; When $\varepsilon=$ 0.25 , public opinion converges around $t=12$, converge into a cluster, all individuals hold the same opinion located in the middle of the opinion space.

When the value of $\varepsilon$ is small, shows that the group has a strong closure, individuals tend to think alone, thus generate isolated nodes, and the smaller the threshold, the more isolated nodes. The value of trust threshold $\varepsilon$ is inversely proportional to the final number of opinion clusters when the evolution of public opinion is stable: With the continuous increase of the trust threshold $\varepsilon$, the time required for group view to stabilize and the final opinion clusters continue to decrease. In this experiment, the relationship between the final opinion clusters and trust threshold $\varepsilon$ have a certain deviation with " $1 / 2 \varepsilon$ criterion" proposed by Weisbuch. Trust threshold can be understood as the distance between individuals in the public opinion dissemination, which reflects the possibility of individual exchange opinion for a social event or phenomenon. The greater the trust threshold, the more likely the group attitude is attributed to consensus.
3.3 The influence of memory length $\mathrm{M}$ on the opinion evolution

Set the number of nodes $\mathrm{N}=1000$, accelerated growth index $\mathrm{r}=1.4$, trust threshold $\varepsilon=0.1$, memory length $\mathrm{M}$ takes 2,5 , respectively. The initial values of all individuals subject to $[0,1]$ uniform distribution. Evolution time $\mathrm{T}=50$, individual opinions are updated synchronously at each time step, experimental results are shown in Figure 2.
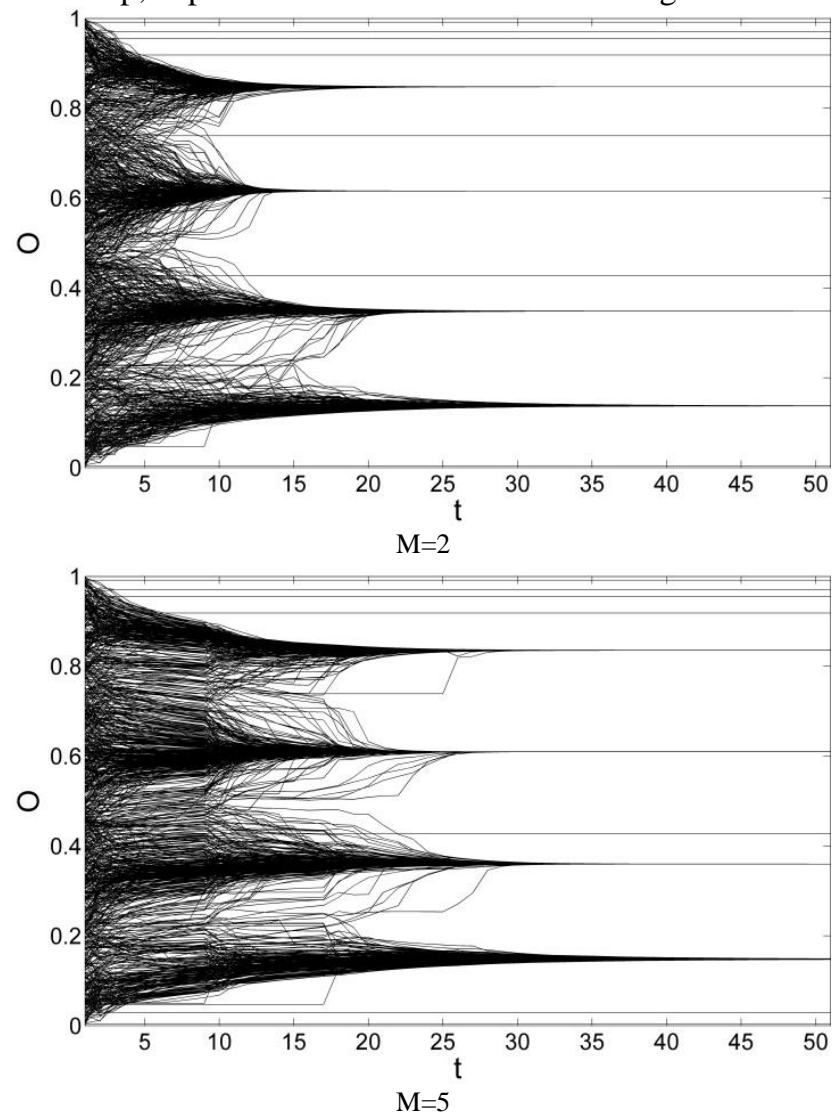

Fig. 2 Effect of memory length $\mathrm{M}$ on public opinion evolution

As shown in figure 2, when the memory length $M=2$, public opinion converges around $t=25$, generating 4 opinion clusters, along with some isolated nodes; when the memory length $\mathrm{M}=5$, public opinion converges around $\mathrm{t}=35$, generating 4 opinion clusters, along with some isolated nodes. When the value of $\mathrm{M}$ is small, public opinions quickly aggregated at the beginning, rapidly forming four clusters; when the value of $\mathrm{M}$ is large, group view is dispersed, public opinion is ladder shaped polymerization, polymerization speed is slow, at the beginning the gap between groups is not obvious, the cluster will be obvious after a certain time.

The results show that the memory length $M$ does not affect the number of clusters after the group attitude is stable, but 
affects the time group attitude tends to be stable, the greater the value of memory length $\mathrm{M}$, the longer the group reached stability. When memory length $\mathrm{M}$ increases, the unity of opinion becomes more difficult, memory hinders the unification of opinion evolution, larger memory length means weaker individual neighbor relationship, interaction between local individuals weakens, and the larger memory length makes it difficult for individuals to change their opinions.

In conclusion, the new model exhibits similar properties to classical finite trust models in the aspect of public opinion evolution, convergence rate and final clusters, but the new model takes into account the influence of user's influence, trust threshold and individual memory strategy on trust relationship, which is more in line with the characteristics of group communication in the actual network environment. At the same time, the new model reproduces the convergence, opposition and differentiation of opinions in public opinion evolution.

\section{Conclusions}

In this paper, the classical limited trust model is improved according to the characteristics of people's opinions interaction in real network, the concept of memory length is introduced and a new method to measure the mutual influence between individuals is proposed. At the same time, considering the accelerated growth of real network, use accelerated growth HK networks as public opinion networks, focus on the interactive effects of trust threshold and memory length on the public opinion evolution.

The experimental results show that the number of opinion clusters and the value of trust threshold are inversely proportional when the group attitude is stable. The smaller the trust threshold, the more opinion clusters. With the increase of the trust threshold, the number of opinion clusters decreases gradually. When the trust threshold exceeds the critical value, the number of opinion clusters is reduced to 1 . This is consistent with the conclusions of the HK model. People only choose what they can touch and what they are interested in, a larger trust threshold means that individuals are more susceptible to the attitudes of neighboring individuals and change attitudes, which makes the public attitude faster consensus. The interaction between local individuals and the weights of neighboring individuals will change after the individual opinion updated. Individual disconnect from the neighbors with big opinion distance in the current memory length, and duplicate these connections at the end of the current memory length. Memory length has a great influence on the stable time of public opinion, the longer the memory length is, the longer the group attitude achieves stability.
Large memory length makes it difficult for individuals to change opinions, and the length of memory hinders the evolution of public opinion.

The research conclusion has certain guiding significance for revealing the evolution law of network public opinion and discussing the effective way of group consistency construction. The deficiency of this paper is that there is no consideration of network tropism and individual heterogeneity, without considering the competition of multiple topics, which are the focus of further research.

\section{Acknowledgments}

In this paper, the research was sponsored by Research on science and technology project of Henan Province (Project No. 152102210277), Project of industry university research cooperation in Henan Province (Project No. 152107000027), Supported by Program for Innovative Research Team (in Science and Technology) in University of Henan Province (Project No.17IRTSTHN010), Technological Innovation Team of Henan University of Science and Technology (Project No.2015XTD011) and Major Cooperative Engagement Fund of Henan University of Science and Technology (Project No.2015ZDCXY03).

\section{References}

[1] Zeng Runxi. Research on Information Resource Sharing of Network Opinion[J]. JOURNAL OF INTELLIGENCE, 2009, 28(8):187-191

[2] SZNAJD-WERON K, SZNAJD J. Opinion evolution in closed community[J]. International Journal of Modern Physics C, 2001, 11(6):1157-1165.

[3] Deffuant G, Neau D, Amblard F, et al. Mixing beliefs among interacting agents[J]. Advances in Complex Systems, 2000, 3(1):87-98.

[4] Hegselmann R, Krause U. Opinion Dynamics and Bounded Confidence Models, Analysis and Simulation[J]. Journal of Artificial Societies \& Social Simulation, 2002, 5(3):2.

[5] Chen Guirong, Cai Wandong, Xu Huijie, etc. High-Effect Priority Bounded Confidence Model for Network Opinion Evolution[J]. Journal of Shanghai Jiaotong University, 2013, 47(1):155-160.

[6] Lu An, Liu Yezheng. Simulation of Group Opinions Evolution Model Based on Continuous Impact Function[J]. Chinese Journal of Management, 2014, 11(2):283-287.

[7] Zhao Yiyi, Peng Yi, Xiao Lei, Li Ling. On opinion propagation mechanism of collective panic buying under emergences[J]. Systems Engineering-Theory \& Practice, 2015, 35(3):616-622.

[8] Su Jiongming, Liu Baohong, Li Qi, Ma Hongxu. Trust, evolution, and consensus of opinions in a social group[J]. Acta Physica Sinica, 2014(5): 36-43.

[9] Cui Aixiang, Fu Yan. Accelerated-growth HK Network Evolution Model[J]. Computer Science, 2015, 42(4):37-39. 
IJCSI International Journal of Computer Science Issues, Volume 14, Issue 1, January 2017

ISSN (Print): 1694-0814 | ISSN (Online): 1694-0784

www.IJCSI.org

Zhang Jingshan, is a Master of Computing Technology, Student. With interest in complex networks, network public opinion.

Ma Baohua, is a Master of Computing Technology, the main research areas are computer network, network public opinion.

Sun Shibao, postdoctoral, Professor, graduate tutor, the main research direction computer networks, digital image processing, etc.

Zhang Yanan, is a Master of Computing Technology, Student. With interest in network public opinion, digital image processing. 